Zusammenfassung. Während der letzten 20 Jahre wurde bei 156 Patienten eine direkte und indirekte Aortenisthmusplastik durchgeführt. Frühkomplikationen traten in 7,6\% auf. Bei 5 Patienten $(3,8 \%)$ entwickelte sich als Spätkomplikation ein Aneurysma im Bereich der Isthmusplastik. Die Gesamtletalität betrug 4,5\%. Kontrolluntersuchungen ergaben keinen Anhalt für funktionell kritische Restenosen. Die von Vossschulte entwickelte Aortenisthmusplastik stellt ein chirurgisch elegantes Verfahren der funktionellen Kontinuitätswiederherstellung mit minimaler Letalität und Restenosegefahr dar.

Schlüsselwörter: Aortenisthmusplastik - Früh- und Spätkomplikationen - Aneurysma.

\title{
181. Tödliche und seltene schwere Verletzungen beim alpinen Skisport
}

\author{
Thomas Hewel, W. R. Mang, P. C. Maurer und Th. Zimmermann \\ Chirurg. Klinik und Orthopädische Klinik des Klinikums rechts der Isar der Technischen Universität \\ München
}

\section{Fatal and Severe Skiing Accidents}

Summary. Twentyseven fatal skiing accidents recorded from 1959-1975 were analyzed without reference to avalanche accidents. The authors' own results were compared with reports of 60 similar skiing injuries. It was found that 44 percent of the fatal accidents were caused by crashing against obstacles. Half of the accidents happened during high-speed skiing or downhill competition. More than two-thirds of the wounded skiers died of head injuries. A suitable head protection is recommended for downhil skiing.

Key words: Accident, fatal: Sport - Injury, head - Collision - Speed.

Zusammenfassung. Untersucht wurden 27 tödliche Unfälle beim Pistenskilauf (ohne Lawinenunglücke) aus den Jahren 1959-1975. Die eigenen Ergebnisse wurden mit Berichten über insgesamt 60 ähnliche Skiverletzungen verglichen. $44 \%$ der tödlichen Skiunfälle waren durch Aufprall gegen Hindernisse verursacht, die Hälfte der Unfälle mit Todesfolge ereignete sich bei erhöhter Geschwindigkeit oder Skirennen. Úber $2 / 3$ aller tödlichen Skiunfälle sind Kopfverletzungen, weshalb die Forderung nach einem geeigneten Kopfschutz beim alpinen Skilauf dringlich und sinnvoll erscheint.

Schlüsselwörter: Tödliche Skiunfälle - Hindernisaufprall - hohe Geschwindigkeit - Kopfverletzungen.

\section{Maligne Melanome: Diagnose, Klassifikation, Therapie}

\author{
H. Bohmert, R. Baumeister, G. Heberer, B.-R. Balda, O. Braun-Falco und B. Konz \\ Chirurgische Klinik und Dermatologische Klinik und Poliklinik der Universität München
}

\section{Malignant Melanomas: Diagnosis, Classification, Therapy}

Summary. Malignant cutaneous melanomas are prognostically variable, histogenetically and diagnostically heterogeneous malignant tumors that stem from pigment-producing cells. Therefore, the therapeutic approach depends on exact differentiation at diagnosis, as to (1) clinical stages: $\mathrm{I}=$ 